\title{
Symbolic Implementation of Preliminary Group Classiffication for Ordinary Differential Equations
}

\author{
K. S. Govinder \\ Astrophysics and Cosmology Research Unit, School of Mathematics, Statistics and Computer Science, University of KwaZulu-Natal, \\ Private Bag X54001, Durban 4001, South Africa
}

Correspondence should be addressed to K. S. Govinder; govinder@ukzn.ac.za

Received 5 May 2013; Accepted 12 August 2013

Academic Editor: Qiankun Song

Copyright (C) 2013 K. S. Govinder. This is an open access article distributed under the Creative Commons Attribution License, which permits unrestricted use, distribution, and reproduction in any medium, provided the original work is properly cited.

We apply the method of preliminary group classification to ordinary differential equations of the form $y^{(n)}=E\left(x, y, y^{\prime}, \ldots, y^{(n-1)}\right.$, $\left.f^{i}(x)\right), i=0, \ldots, m$. We show how this method can be implemented via the popular symmetry analysis package PROGRAM LIE provided that the package is supplemented with a small file. We then apply this method to an extension of the generalised EmdenFowler equation given by $y^{\prime \prime}=\sum_{i=2}^{5} f^{i}(x) y^{i}$.

\section{Introduction}

Lie symmetry analysis $[1-4]$ is a useful tool in the analysis of differential equations. It often provides routes to solutions where none (or few) exist in addition to usually providing a basis for other ad hoc approaches. A major disadvantage of the Lie approach was that it was quite cumbersome-one had to wade through a system of linear partial differential equations in order to find the symmetries (see [5] for an example of the complexity of the equations) before actually being able to use the symmetries themselves. Fortunately, there are a number of packages available today $[6,7]$ which remove this obstacle. We have used SYM [8] and PROGRAM LIE [9] to good effect. We mention PROGRAM LIE in particular as it provides results when many other (newer) packages fail.

In the event that the equation being analysed includes arbitrary functions, the analysis usually has to be completed manually. One ends up with a differential equation for the arbitrary function, and one has to analyse this equation to determine the possible forms of the function which will lead to additional symmetries. However, this approach is largely dependent on the perspicacity and persistence of the practitioner; it is easy to omit relevant cases [10]. Fortunately, the method of preliminary group classification [11, 12] can be used to good effect in such situations. However, again, this method must largely be completed manually. While this is a partial classification, it has the advantage of involving algebraic manipulations and not the solution of differential equations. Here, we discuss the method and show how, in the case of ordinary differential equations where the arbitrary function depends only on the independent variable, one can implement the method computationally. We apply the method to an important equation in mathematical physics.

\section{Preliminary Group Classification}

In order to find the point symmetries of the $n$th order ordinary differential equation

$$
y^{(n)}=E\left(x, y, y^{\prime}, \ldots, y^{(n-1)}, f^{i}(x)\right), \quad i=0, \ldots, m,
$$

where the $f^{i}$ are arbitrary functions of $x$, we search for an operator of the form

$$
U=\xi(x, y) \frac{\partial}{\partial x}+\eta(x, y) \frac{\partial}{\partial y}
$$

that leaves (1) invariant; that is, we require

$$
\left.U^{[n]}(1)\right|_{(1)}=0 .
$$

In (3), $U^{[n]}$ is the $n$th prolongation of $U$ given by [13]

$$
U^{[n]}=U+\sum_{i=1}^{n}\left\{\eta^{(i)}-\sum_{j=1}^{i}\left(\begin{array}{l}
i \\
j
\end{array}\right) y^{(i+1-j)} \xi^{(j)}\right\} \frac{\partial}{\partial y^{(i)}},
$$


where the superscripts refer to total differentiation with respect to $x$; that is,

$$
\begin{gathered}
\eta^{\prime}=D_{x} \eta, \\
D_{x}=\frac{\partial}{\partial x}+y^{\prime} \frac{\partial}{\partial y}+\cdots,
\end{gathered}
$$

and so on. Note also that (3) must take the equation into account (which is the reason for the subscript (1)).

The result of (1) is a partial differential equation in $\xi$ and $\eta$ (and the $f^{i}(x)$ ). Separating by coefficients of functions of $y^{(n-1)}$ (and then lower derivatives) results in an overdetermined system of linear partial differential equations. This system is then reduced to equations for the $f^{i}(x)$. Equation (1) will admit symmetries contingent on the solution of the equations for the $f^{i}(x)$.

In the case of the preliminary group classification, on the other hand, we look for operators of the form

$$
X=\xi(x, y) \frac{\partial}{\partial x}+\eta(x, y) \frac{\partial}{\partial y}+\omega_{i}\left(x, y, f^{i}\right) \frac{\partial}{\partial f^{i}},
$$

where we have used the Einstein summation convention over repeated indices. Note that while $\xi$ and $\eta$ are functions in $(x, y)$ space, the $\omega_{i}$ are functions in $\left(x, y, f^{i}\right)$ space-the $f^{i}$ are now treated as differentiable variables. This operator generates an equivalence transformation of (1) if and only if [11]

$$
\begin{gathered}
\left.\widetilde{X}\left(y^{(n)}-E\left(x, y, y^{\prime}, \ldots, y^{(n-1)}, f^{i}\right)\right)\right|_{y^{(n)}=E\left(x, y, y^{\prime}, \ldots, y^{(n-1)}, f^{i}\right)}=0, \\
\widetilde{X}\left(f_{y}^{i}\right)=0 .
\end{gathered}
$$

Note that we have supplemented (1) with

$$
f_{y}^{i}=0
$$

as the $f^{i}$ only depend on $x$.

Now, we define $\widetilde{X}$ as the appropriate prolongation of $X$ defined via (4) but with the following modification:

$$
\widetilde{X}=\left.X^{[n]}\right|_{\omega_{i}=0}+\omega_{i}\left(x, y, f^{i}\right) \frac{\partial}{\partial f^{i}}+\omega_{i}^{\prime} \frac{\partial}{\partial f_{y}^{i}},
$$

where

$$
\begin{gathered}
\omega_{i}^{\prime}=\widetilde{D}_{y} \omega_{i}-f_{x}^{i} \widetilde{D}_{y} \xi \\
\widetilde{D}_{y}=\frac{\partial}{\partial y}+f_{y}^{i} \frac{\partial}{\partial f^{i}}=\frac{\partial}{\partial y} .
\end{gathered}
$$

Regardless of the form of (1), condition (8) requires

$$
\omega_{i}^{\prime}=0 .
$$

For this to hold for arbitrary $f^{i}$, we must have (from (11))

$$
\frac{\partial \omega_{i}}{\partial y}=\xi_{y}=0
$$

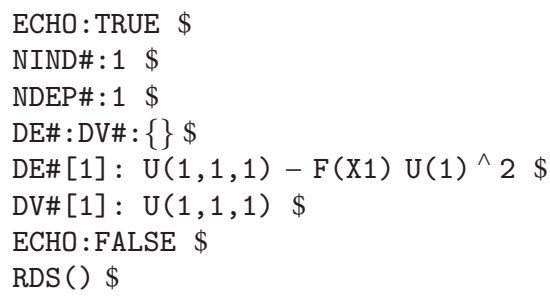

Algorithm 1

and so, in general, we have

$$
\xi=\xi(x) \quad \eta=\eta(x, y) \quad \omega_{i}=\omega_{i}\left(x, f^{i}\right) .
$$

In other words, when we look for equivalence transformations of (1), we seek operators of the form

$$
U=\xi(x) \frac{\partial}{\partial x}+\eta(x, y) \frac{\partial}{\partial y}+\omega_{i}\left(x, f^{i}\right) \frac{\partial}{\partial f^{i}},
$$

and then require

$$
\left.U^{[n]}(1)\right|_{(1)}=0,
$$

where $U^{[n]}$ is given by (4) (in fact, the arguments of $\omega_{i}$ will always be the $f^{i}$ and the functional dependent of the $f^{i}$ ). Note that (17) is the standard condition that most computer packages invoked to find symmetries of differential equations. However, by ensuring that our coefficient functions are of the form (15), we are actually finding equivalence transformations.

\section{Implementation in PROGRAM LIE}

The full details of using PROGRAM LIE can be found in [9] as well as the files lie.doc, morelie.doc, and readme.lie included with the distribution, so we will not go into the details here. We merely show how to implement the requirement (15) using the Emden-Fowler equation

$$
y^{\prime \prime}=f(x) y^{2} .
$$

This equation (and variations thereof) has attracted enormous interest over the years. It arises in a number of applications, not the least being in the study of shear-free spherically symmetric spacetimes in general relativity $[12,14-$ 23] (see [24] for a survey up to 1975 which, even then, had numerous references). Variations of (18) can be found in the study of stellar structure [25], thermodynamics [26], and other fields [27].

The file usually created to analyse this equation for point symmetries is given by Algorithm 1 .

However, in the method of preliminary group classification, the arbitrary function $f(x)$ is treated as a variable. Thus, instead of one dependent variable, we will have two. As a result, the input file is modified to read as Algorithm 2. 


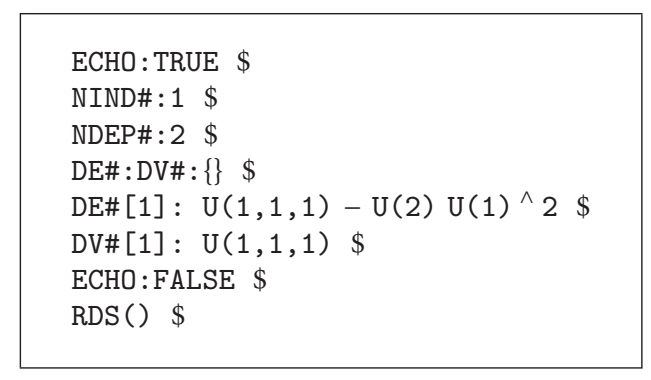

Algorithm 2

A normal run of PROGRAM LIE for (18) will require entering the command RDS(EF1,DAT); at the ? prompt (where we have assumed that the input file has been named EF1.DAT). Thereafter, one enters DOLIE ();. Now, we examine the equations using the command A\#; (which is usually not necessary in the standard analysis). This outputs the equations to be analysed to the screen. In this case, the output is Algorithm 3.

The final three lines of output indicate the coeffiient fun ctions with the correspondence $\xi \rightarrow \mathrm{F} \#(3, \mathrm{U} 1, \mathrm{X} 1)+, \eta \rightarrow$ $\mathrm{F} \#(1, \mathrm{U} 1, \mathrm{X} 1)$ and $\omega \rightarrow \mathrm{F} \#(2, \mathrm{U} 1, \mathrm{U} 2, \mathrm{X} 1)$. In order to implement the requirement (15), we need to enter, in turn, the following three commands at the ? prompt (see Algorithm 4).

Continuing the programme via $\operatorname{DOSOLV}()$; $\operatorname{DOCHECK}()$; , and then $\operatorname{DOVEC}()$; will produce the required equivalence transformations.

Instead of entering these steps in manually, all one needs to do, after reading in the file name via $\mathrm{RDS}(\mathrm{EF} 1, \mathrm{DAT})$; is to enter the command RDS (PGC,FIX); where pgc.fix is given in Algorithm 5.

\section{Application to an Extended Emden-Fowler-Type Equation}

A simple extension of (18), namely,

$$
y^{\prime \prime}=\sum_{i=2}^{5} f^{i}(x) y^{i}
$$

was analysed by Leach and Maharaj [28]. In the case of $f^{i}(x)=0, i=3 \cdots 5$, it reduces to (18). If we now relax the requirement to $f^{i}(x) \neq 0$, the equation is central in the study of charged shear-free spherically symmetric spacetimes [15-17, 29-31]. Leach and Maharaj [28] analyse this general equation (19) and interpret it as a time-dependent anharmonic oscillator. We observe that the only case of (19) being linearisable via a point transformation is when it is linear, that is, when $f^{i}(x)=0, i=2 \cdots 5$.

While a variety of methods have been used in the analysis of restricted forms of (19), the most successful method is that of Lie's theory of extended groups. In most cases, (except for [12] where the case of (18) was analysed), the method has been applied directly $[19,20]$; that is, (19) (or subcases thereof) was assumed to possess a Lie point symmetry of the form (2). Here we apply the systematic method of preliminary group classification utilising pgc.fix in PROGRAM LIE. As we have more than one unknown function, we need to modify pgc.fix to allow for additional functions. All we need do is repeat the line A\#: EVSUB (A\#, F\# (2, U1, U2, X1), F\# (2000, U2, X1)); until we have covered all the functions (in our case we have four functions) and then increment the line A\#: $\operatorname{EVSUB}(A \#, F \#(3, \mathrm{U} 1, \mathrm{U} 2, \mathrm{X} 1), \mathrm{F} \#(3000, \mathrm{X} 1))$; appropriately.

We find the following four equivalence transformations for (19):

$$
\begin{aligned}
& X_{1}=x^{2} \frac{\partial}{\partial x}+x y \frac{\partial}{\partial y}-(i+3) x f^{i} \frac{\partial}{\partial f^{i}}, \\
& X_{2}=-x \frac{\partial}{\partial x}-\frac{y}{2} \frac{\partial}{\partial y}+\frac{i+3}{2} f^{i} \frac{\partial}{\partial f^{i}}, \\
& X_{3}=-\frac{\partial}{\partial x}, \\
& X_{4}=y \frac{\partial}{\partial y}-(i-1) f^{i} \frac{\partial}{\partial f^{i}},
\end{aligned}
$$

with Lie algebra $L_{X}$ given by Lie bracket relationships

$$
\left[X_{1}, X_{2}\right]=X_{1} \quad\left[X_{2}, X_{3}\right]=X_{3} \quad\left[X_{3}, X_{1}\right]=2 X_{2} .
$$

This corresponds to $A_{3,8} \oplus A_{1}$ in [32].

As we are interested in functional forms for $f^{i}$ (all of which depend only on $x$ ), we need to consider projections of (20) onto the $\left(x, f^{i}\right)$ space; that is, we utilise the Lie algebra $L_{G}$ comprised of the operators

$$
\begin{aligned}
& G_{1}=x^{2} \frac{\partial}{\partial x}-(i+3) x f^{i} \frac{\partial}{\partial f^{i}} \\
& G_{2}=-x \frac{\partial}{\partial x}+\frac{i+3}{2} f^{i} \frac{\partial}{\partial f^{i}} \\
& G_{3}=-\frac{\partial}{\partial x} \\
& G_{4}=-(i-1) f^{i} \frac{\partial}{\partial f^{i}} .
\end{aligned}
$$

The main ideas of the method of preliminary group classification reside in the following facts [11]: (i) if $f^{i}=F^{i}(x)$ is invariant with respect to an element of $L_{G}$, then (19) is invariant with respect to the corresponding element of $L_{X}$, and (ii) all equations of the form

$$
y^{\prime \prime}=\sum_{i=2}^{5} \widetilde{f}^{i}(x),
$$

which admit similar Lie algebras which are subalgebras of $L_{G}$, must be equivalent with respect to the equivalence transformation obtained via $L_{X}$. (While we have stated these conditions with regard to the specific equation (19) and Lie algebras $L_{X}$ and $L_{G}$, they are applicable in a more general 


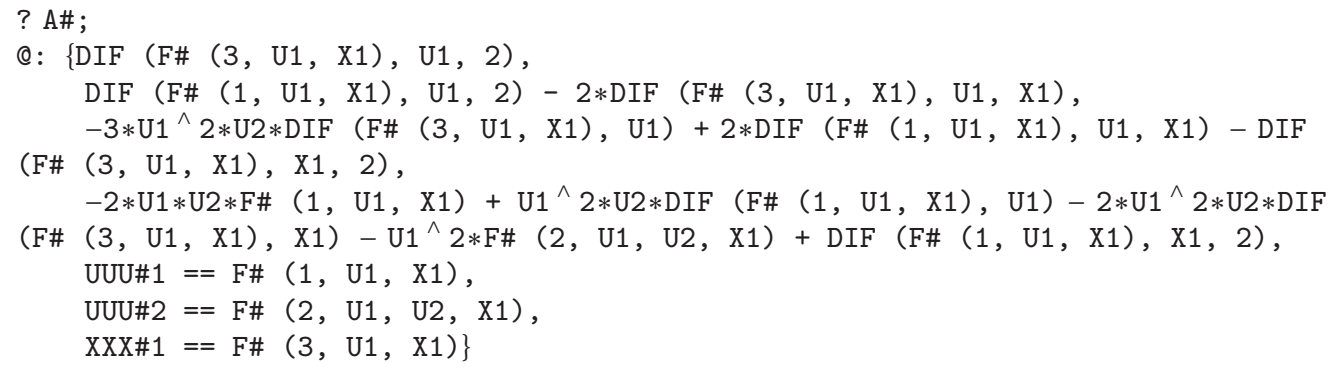

Algorithm 3

TABLE 1: Invariant forms of $f^{i}$ admitted by the equivalence transformations of (19) with $\lambda_{i}$ arbitrary constants.

\begin{tabular}{lcr}
\hline Operator & Invariant form of $f^{i}$ & $f^{2}$ \\
\hline$G_{1}$ & $\lambda_{i} x^{-(i+3)}$ & $\lambda_{2} x^{-5}$ \\
$G_{4}$ & Null & $\lambda_{2} x^{\alpha-(5 / 2)}$ \\
$G_{2}+\alpha G_{4}$ & $\lambda_{i} x^{(\alpha-(1 / 2)) i-(\alpha+(3 / 2))}$ & $\lambda_{2}(x-1)^{-(\beta+5) / 2}(x+1)^{(\beta-5) / 2}$ \\
$G_{1}+G_{3}+\beta G_{4}$ & $\lambda_{i}(x-1)^{(-(1+\beta) i+\beta-3) / 2}(x+1)^{((\beta-1) i-\beta-3) / 2}$ & $\lambda_{2} x^{-5} \exp (\varepsilon / x)$ \\
$G_{1}+\varepsilon G_{4}$ & $\lambda_{i} x^{-(i+3)} \exp (\varepsilon(i-1) / x)$ & \\
\hline
\end{tabular}

EVSA\#(F\#(1,U1, U2, X1), F\#(1000,U1, X1));

$\operatorname{EVSA\# }(\mathrm{F} \#(2, \mathrm{U} 1, \mathrm{U} 2, \mathrm{X} 1), \mathrm{F} \#(2000, \mathrm{U} 2, \mathrm{X} 1))$;

EVSA\# $(F \#(3, \mathrm{U} 1, \mathrm{X} 1), \mathrm{F} \#(3000, \mathrm{X} 1))$;

Algorithm 4

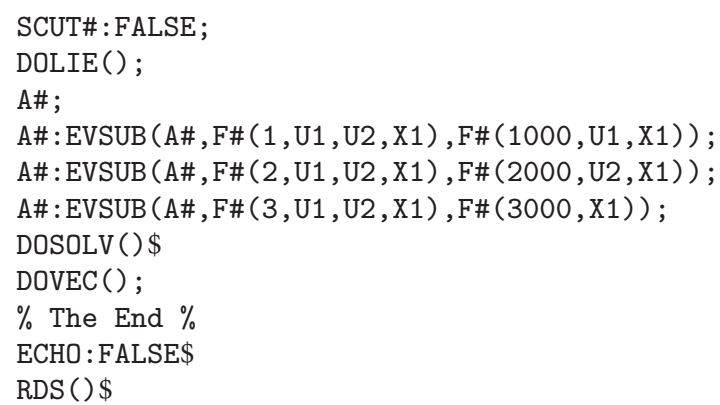

Algorithm 5

setting.) We use these facts to determine those forms of $f^{i}$ for which (19) admits symmetries.

The subalgebras of low-dimensional Lie algebras have already been determined by Patera and Winternitz [32] (though there are some concerns over the accuracy of that work [10]). For $A_{3,8} \oplus A_{1}$, the one-dimensional subalgebras are given by

$$
\left\langle G_{1}\right\rangle ;\left\langle G_{4}\right\rangle ;\left\langle G_{2}+\alpha G_{4}\right\rangle ;\left\langle G_{1}+G_{3}+\beta G_{4}\right\rangle ;\left\langle G_{1}+\varepsilon G_{4}\right\rangle
$$

where $\alpha \geq 0, \beta$ is arbitrary, and $\varepsilon= \pm 1$. The forms of $f^{i}$ which allow for a symmetry (after projection onto the $(x, y)$ space) of the form given above are given by calculating the invariants of each equivalence transformation. The results are given in Table 1 . We also give the results for the $i=2$ case given the enormous interest in this particular form of the equation.

Remark 1. In Table 1, we have all the cases for which (19) admits a single symmetry. What this means is that all other cases of (19) which admit a single symmetry can be transformed to one of the cases listed here. Importantly, the one case listed in [28] but missing from this table is that of $f^{i}=\lambda_{i} \exp ((i-1) x)$. However, this case is simply obtained by the combination $G_{3}+G_{4}$. It is a simple matter to transform this form of $f^{i}$ to the result in the final row of Table 1 . However, we note that the result contained in that row has not previously been found.

In [28], Leach and Maharaj provide a detailed Lie analysis of (19) including forms for $f^{i}$ which admit one or two symmetries. Here, we have been able to reproduce their results through a simple calculation and utilising a computer package. Interestingly, the forms of $f^{i}$ which admit two symmetries are easy to find, again without resorting to the laborious calculations in [28]. Indeed, all one needs to do is look at the two-dimensional subalgebras of $A_{3,8} \oplus A_{1}$ [32] as follows:

$$
\left\langle G_{1}, G_{4}\right\rangle ;\left\langle G_{2}, G_{4}\right\rangle ;\left\langle G_{1}+G_{3}, G_{4}\right\rangle ;\left\langle G_{2}+\alpha G_{4}, G_{1}\right\rangle,
$$

where $\alpha$ is arbitrary. Since $G_{4}$ does not affect the invariant form of the $f^{i}$, any invariant form generated by $G_{1}$ and $G_{2}$ would be unaffected by the operation of $G_{4}$. Thus, the $f^{i}$ in the first row of Table 1 and the third row (with $\alpha=0$ ) will 
have $X_{4}$ as a second symmetry. This takes care of the first two cases in (25). In fact, this applies to the third case as well if we take $\beta=0$ in the fourth row of Table 1. Finally, we have the fourth case of (25). Here, we take the result of the third row in Table 1 and operate on it with $G_{1}$. The result forces $\alpha=-(i+3) /(2(i-1))$, and the invariant form of $f^{i}$ reduces to that in the first row. These results correspond to the three cases in [28] which admit two symmetries (up to scalings and translations). We are thus able to provide all the results of [28] in a straightforward, algorithmic manner.

\section{Discussion}

We have been able to provide a simple means of automating the search for (restricted) equivalence transformations for ordinary differential equations which have arbitrary functions of the independent variable. This was achieved by supplementing PROGRAM LIE [9] with some simple substitution commands based on results from the theory. We were able to apply this approach to an important equation in mathematical physics. All known results were regenerated in a straightforward, uncomplicated fashion.

This approach will also work for partial differential equations. Again, the only class of equations for which the search for equivalence transformations can be automated is that which contains arbitrary functions of the independent variable. A similar fix to pgc.fix will work in those cases.

\section{Acknowledgments}

The authors thank the University of KwaZulu-Natal and the National Research Foundation of South Africa for continuing support. This paper was completed while visiting the Mathematical Biosciences Institute at Ohio State University in Columbus, Ohio, USA, and they thank Professor M. Golubitsky and the staff of the institute for their warm hospitality and support during their stay. They also thank Michael Okelola for providing them with a copy of [12].

\section{References}

[1] G. W. Bluman and S. Kumei, Symmetries and Differential Equations, vol. 81 of Applied Mathematical Sciences, Springer, New York, NY, USA, 1989.

[2] P. J. Olver, Applications of Lie Groups to Differential Equations, vol. 107 of Graduate Texts in Mathematics, Springer, New York, NY, USA, 2nd edition, 1993.

[3] H. Stephani, Differential Equations. Their Solution Using Symmetries, Cambridge University Press, Cambridge, UK, 1989.

[4] L. E. Dickson, "Differential equations from the group standpoint," Annals of Mathematics, vol. 25, pp. 287-378, 1925.

[5] P. G. L. Leach and V. M. Gorringe, "The relationship between the symmetries of and the existence of conserved vectors for the equation $\ddot{\mathrm{r}}+f(r) \mathrm{L}+g(r) \mathrm{r}=0$," Journal of Physics A, vol. 23, no. 13 , pp. 2765-2774, 1990.

[6] W. Hereman, "Review of symbolic software for Lie symmetry analysis," Mathematical and Computer Modelling, vol. 25, no. 8-9, pp. 115-132, 1997, Algorithms and software for symbolic analysis of nonlinear systems.
[7] J. Butcher, J. Carminati, and K. T. Vu, "A comparative study of some computer algebra packages which determine the Lie point symmetries of differential equations," Computer Physics Communications, vol. 155, no. 2, pp. 92-114, 2003.

[8] S. Dimas and D. Tsoubelis, "A new symmetry-finding package for mathematica," in Proceedings of the 10th International Conference in Modern Group Analysis, N. H. Ibragimov, C. Sophocleous, and P. A. Pantelis, Eds., pp. 64-70, University of Cyprus, Larnaca, Cyprus, 2005.

[9] A. K. Head, "LIE, a PC program for Lie analysis of differential equations," Computer Physics Communications, vol. 77, no. 2, pp. 241-248, 1993.

[10] R. M. Edelstein and K. S. Govinder, "On a preliminary group classification of the nonlinear heat conduction equation," Quaestiones Mathematicae, vol. 31, no. 3, pp. 225-240, 2008.

[11] N. H. Ibragimov, M. Torrisi, and A. Valenti, "Preliminary group classification of equations $v_{t t}=f\left(x, v_{x}\right) v_{x x}+g\left(x, v_{x}\right)$," Journal of Mathematical Physics, vol. 32, no. 11, pp. 2988-2995, 1991.

[12] C. W. Soh and F. M. Mahomed, "Preliminary group classification of $y^{\prime \prime}=f(x) y^{2}$," Mathematical and Computational Applications, vol. 4, no. 1, pp. 289-295, 1999.

[13] F. M. Mahomed and P. G. L. Leach, "Symmetry Lie algebras of $n$th order ordinary differential equations," Journal of Mathematical Analysis and Applications, vol. 151, no. 1, pp. 80-107, 1990.

[14] M. Wyman, "Jeffrey-Williams lecture: nonstatic radially symmetric distributions of matter," Canadian Mathematical Bulletin, vol. 19, p. 343, 1976.

[15] R. A. Sussman, "On spherically symmetric shear-free perfect fluid configurations (neutral and charged). I," Journal of Mathematical Physics, vol. 28, pp. 1118-1136, 1987.

[16] R. A. Sussman, "On spherically symmetric shear-free perfect fluid configurations (neutral and charged). II," Journal of Mathematical Physics, vol. 29, pp. 945-970, 1988.

[17] R. A. Sussman, "On spherically symmetric shear-free perfect fluid configurations (neutral and charged). III," Journal of Mathematical Physics, vol. 29, no. 5, pp. 1177-1211, 1987.

[18] D. C. Srivastava, "Exact solutions for shear-free motion of spherically symmetric perfect fluid distributions in general relativity," Classical and Quantum Gravity, vol. 4, no. 5, pp. 1093-1117, 1987.

[19] P. G. L. Leach, R. Maartens, and S. D. Maharaj, "Self-similar solutions of the generalized Emden-Fowler equation," International Journal of Non-Linear Mechanics, vol. 27, no. 4, pp. 575$582,1992$.

[20] S. D. Maharaj, P. G. L. Leach, and R. Maartens, "Expanding spherically symmetric models without shear," General Relativity and Gravitation, vol. 28, no. 1, pp. 35-50, 1996.

[21] V. K. Chandrasekar, M. Senthilvelan, and M. Lakshmanan, "On the complete integrability and linearization of certain secondorder nonlinear ordinary differential equations," Proceedings of The Royal Society of London A, vol. 461, no. 2060, pp. 2451-2477, 2005.

[22] V. K. Chandrasekar, M. Senthilvelan, and M. Lakshmanan, "On the general solution for the modified Emden-type equation $\ddot{x}+$ $\alpha x \dot{x}+\beta x^{3}=0$," Journal of Physics A, vol. 40, no. 18, pp. 47174727, 2007.

[23] M. C. Kweyama, K. S. Govinder, and S. D. Maharaj, "Noether and Lie symmetries for charged perfect fluids," Classical and Quantum Gravity, vol. 28, no. 10, Article ID 105005, 15 pages, 2011. 
[24] J. S. W. Wong, "On the generalized Emden-Fowler equation," SIAM Review, vol. 17, no. 2, pp. 339-360, 1975.

[25] S. Chandrasekhar, An Introduction to the Study of Stellar Structure, Dover, New York, NY, USA, 1957.

[26] H. T. Davis, Introduction to Nonlinear Differential and Integral Equations, Dover, New York, NY, USA, 1962.

[27] K. Parand, S. Abbasbandy, S. Kazem, and A. R. Rezaei, "An improved numerical method for a class of astrophysics problems based on radial basis functions," Physica Scripta, vol. 83, no. 1, Article ID 015011, 2011.

[28] P. G. L. Leach and S. D. Maharaj, "A first integral for a class of time-dependent anharmonic oscillators with multiple anharmonicities," Journal of Mathematical Physics, vol. 33, no. 6, pp. 2023-2030, 1992.

[29] D. C. Srivastava, "Exact solutions for shear-free motion of spherically symmetric charged perfect fluid distributions in general relativity," Fortschritte der Physik, vol. 40, no. 1, pp. 3172, 1992.

[30] R. Halburd, "Solvable models of relativistic charged spherically symmetric fluids," Classical and Quantum Gravity, vol. 18, no. 1, pp. 11-25, 2001.

[31] C. W. Soh and F. M. Mahomed, "Non-static shear-free spherically symmetric charged perfect fluid distributions: a symmetry approach," Classical and Quantum Gravity, vol. 17, no. 15, pp. 3063-3072, 2000.

[32] J. Patera and P. Winternitz, "Subalgebras of real three- and fourdimensional Lie algebras," Journal of Mathematical Physics, vol. 18, no. 7, pp. 1449-1455, 1977. 


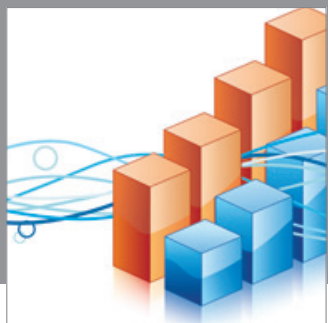

Advances in

Operations Research

mansans

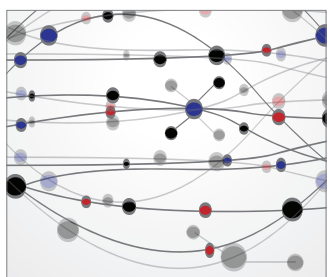

The Scientific World Journal
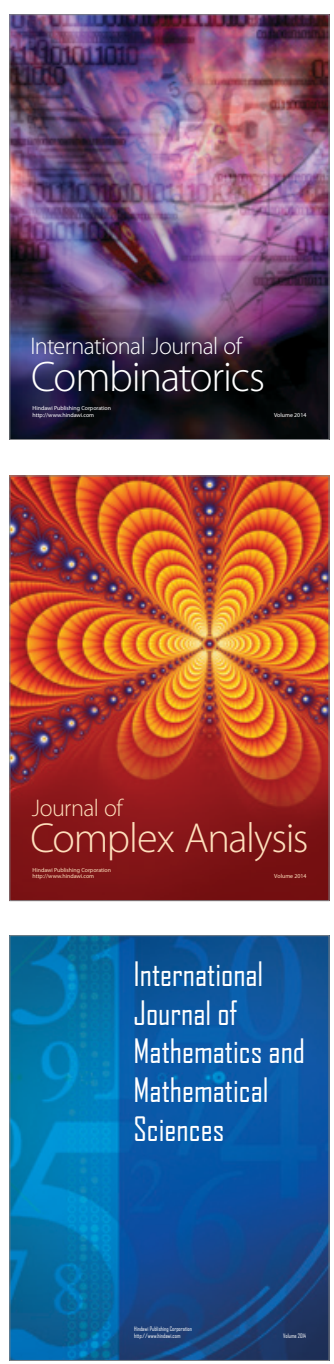
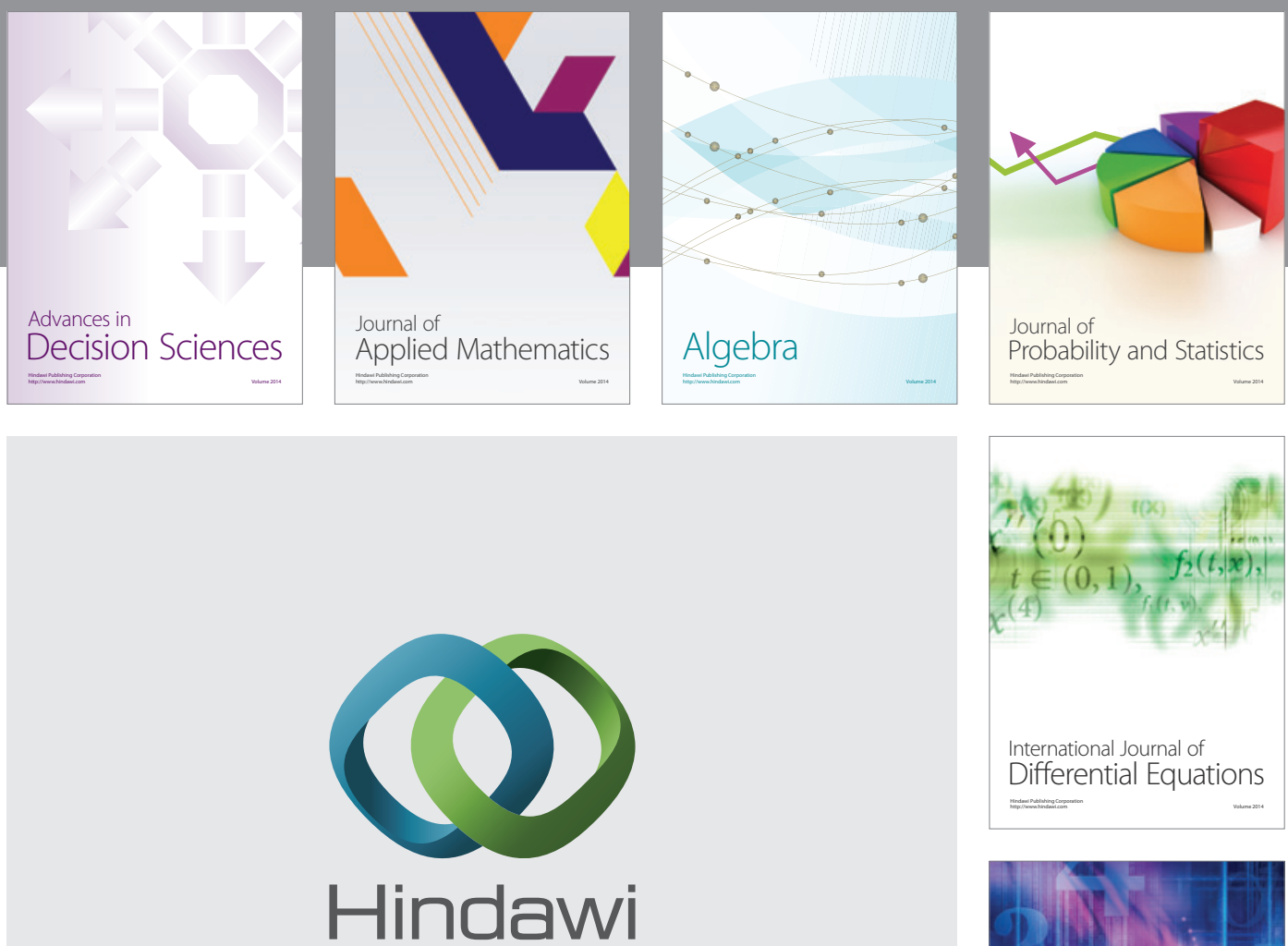

Submit your manuscripts at http://www.hindawi.com
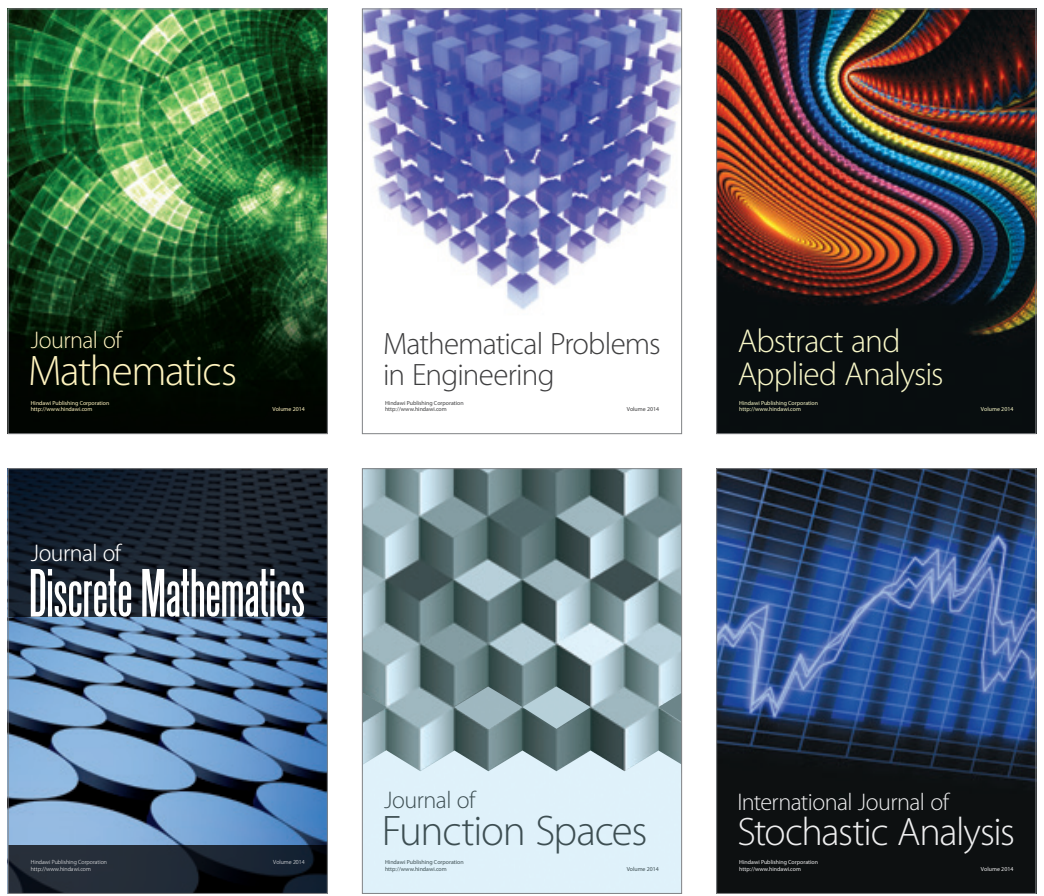

Journal of

Function Spaces

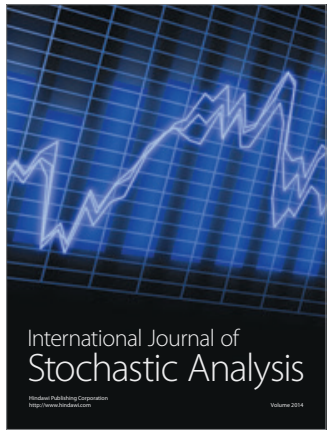

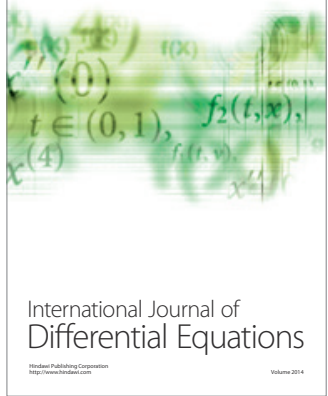
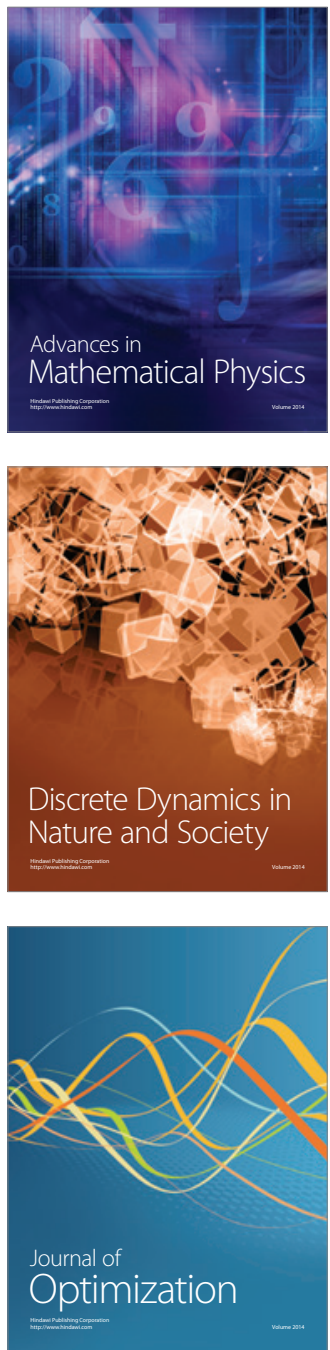